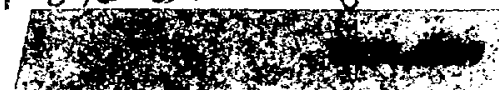

UCRL--96329

DE87 010814

\title{
Optical Guiding and Beam Bending in Free-Electron Lasers
}

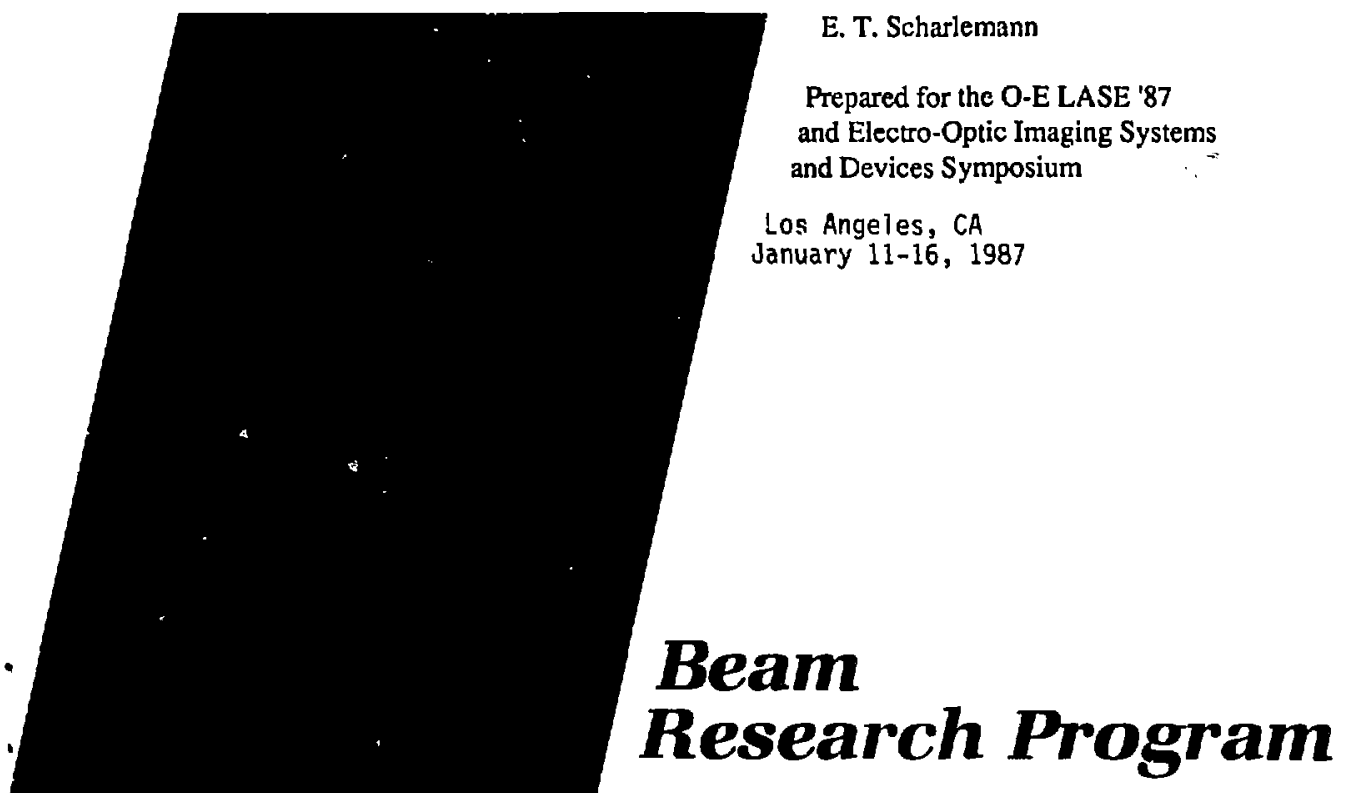


DISCI.AIMIR

This document was presared as an account of work sponsored by an anency of the Inlied Sinles Government. Nelther the Uniled Siates Government nor the Univraliy of Califorala net any of their employees, makes any whrranty, exprets of implied, of assumes any letal thabilliy of responsibillity for the accurucy, completentsh or usefal. acss of any informullow, apperatuse producl, of process diselosed, or represnis that IIs use nowh not biltinge privately onned rights. Keference herein to any smeclit conmercial preducts, proces, or servle! by Irade name, trademark. manufacturer, or othe inise, does and necessarily constilute or imply itg endorsement, recommendation. or tevoring by the Unied Siates Guvernment of the University of Callionala. The views and upintons of awhors expressed herein do wot mesessarily state or mefect these of the United States Government or the University of Californila, and aball not to esed for advertising or produrl emdorsenent morposes. 
Optical guiding and beam bending in free-eleciron lasers

E. T. Scharlemann

Lawrence Livermore liational Laboratory

LIvermore, California

\section{Abstract}

The electron beam in a free-electron laser (FEL) can act as an optical fiber, guiding or bending the optical beam. The refractive and gain effects of the bunched electron beam can compensate for diffraction, making possible wigglers that are many Rayleigh ranges il.e., characteristic diffraction lengths) long.

The origin of optical gulding can be understood by examining gain and refractive guiding in a flber with a complex index of refraction, providing a mathematical description applicable also to the FEL, with some extensions. In the exponential gain regime of the FEL, the electron equations of motion must be included, but a self-consistent description of exponential gain with diffraction fully included becomes possible.

The origin of the effective index of refraction of an FEL is 11lustrated with a simple example of bunched, radiating dipoles. Some of the properties of the index of refraction are described.

The limited experimental evidence for optical beam bending is summarized. The evidence does not yet provide conclusive proof of the existence of optical guiding, but supports the idea.

Finally, the importance of refractive guiding for the performance of a high-gain tapered-wiggler FEL amplifier is lllustrated with numerical simulations.

Work performed jointly under the auspices of the U.S. Department of Energy under W-7405-ENG-48 and for the Department of Defense under SOIO/BMD-ATC MIPR NO. W3-RPD-53-A127.

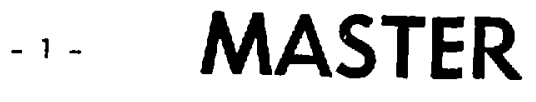




\section{Introduction}

Many free-electron laser applications--for high gain at short wavelengths or high extraction with a tapered wiggler amplifier--require a very long wiggler. If the wiggler must be much longer than the characteristic diffraction length (the Rayleigh range) of the light, diffraction of the light away from the electron beam becomes a critical issue. There is (in theory, at least) an effect that prevents diffraction--the bunched electron beam, at high enough current, can behave as an optical fiber and guide the light. The effect is illustrated in Fig. 1, which is a plot, from a numerical simulation, of light intensity vs horizontal position ( $x$ ) and distance along the wiggler $(z)$; the intensity remains strongly peaked around the electron beam (radius $0.26 \mathrm{~cm})$ throughout the wiggler. The simulation paramete:s for Fig. I, and other figures in this paper, are given in Table 1; the simulations themselves were made with FRED, the LLNL FEL simulation code. ${ }^{1,2,3}$ optical guiding, a) though very important for the successful operation of long-wiggler FELs, has not yet been demonstrated experimentaly.

The Rayleigh range is defined by

$$
z_{R}=\frac{\pi w_{0}^{2}}{\lambda}
$$

and appears in diffraction theory through, for example, the behavior of the spot size $w$ (a radius to the $1 / e^{2}$ intensity point) of a Gaussian TEM 00 mode :

$$
w=w_{0}\left(1+\frac{z^{2}}{z_{R}^{2}}, 1 / 2 \simeq \frac{w_{0} z}{z_{R}},\right.
$$


where the last approximate expression holds for $z \gg z_{R}$. If the on-axis intensity of light in a wiggler were determined by diffraction alone, the wiggler would be restricted to $\leq 3-4 Z_{R}$. Good coupling to the electron beam would also require that the light spot size $w$ be approximately the electron beam size; the length restriction of $3-4 z_{R}$ would thein be a short physical length, severely limiting the possible extraction efficiency (or gain) of the ampilifier.

Two phenomena can counteract diffraction and keep the light confined to near the electron beam over many Rayleigh ranges. The first is galn guiding, described in some detall for a quadratic gain medium in Ref. 4 , and having the very simple explanation that light tends to be where it is amplified. In a gain.guiding medium, the intrinsic gain lenith is shorter than or comparable to the Rayleigh length of the equilibrium gain-guided profile, and the virtual focus (the point at the center of the radius of curvature of the phase fronts) is of order one Rayleigh range behind any point of oloservation.

The second phenomenon is less well known, and is nearly unlque to the FEL. We have referred to it as refractive guiding; it involves the phase shift ( $v_{\text {phase }}(c)$ of light in an FEL. The phase stift, and refractive guiding, can be independent of the gain, and may occur even in the absence of gain (e.g., after saturation in an untapered wiggler). This phenomenon is gerierally less well appreciated than gain guiding, because in a conventional laser medium governed by the Kramers-Kronig relation, the real part of the index of refraction (hence the linear phase shift) vanishes at peak gain; at the design point of the laser mediun, therefore, refractive guiding necessarily vanishes. The relevance of the Kramers-Kronig relations to refractive guiding in an FEL will be discussed below (Sec. VI). 
The significance of the phase shift of the light in an FEL (or the real part of an effective index of refraction) was realized nearly simultaneously by Slater and Lowentha $1,{ }^{5}$ Kroll, Morton, and Rosenbluth ${ }^{6}$ (KMR), Prosnitz, Szoke and Neil, ${ }^{7}$ and Sprangle and Tang. ${ }^{8}$ The significant feature of refractive guiding--that wigglers much longer than a few Rayleigh ranges become possible--was first unambiguously pointed out by KMR, who refer to "optical beam trapping, which could be helpful for devices with $L>k r_{e}^{2}$." Their $r_{e}$ is the electron beam radius, so that $k r_{e}^{2}$ is twice the Rayleigh range obtalned by equating the optlcal mode size to the electron beam size, a reasonable approximation for a high gain amplifler. Although the effect has often been scen in numerical simulations, $5,8,9$ very little analysis of refractive or gain guiding (jointly referred to here as optical guiding) was done unt1l recently when Moore ${ }^{10}$ realized that the exponential gain regime of an $\mathrm{FEL}$, in the presence of arbitrarlly strong diffraction, could be treated nearly exactly--with only the approximation that the light profile propagated self-similarly along with the electron beam. Similar conclusions were independently obtained from a useful analogy to optical fibers by others. "Since the appearance of these two papers there has been considerable theoretical analysis of optical guiding, primarily gain gulding, inspired by the possibility of actually bullding devices with long wigglers. ${ }^{12,13}$

Little experimental evidence for optical guiding in an FEL yet exists; the possible exception is a phenomenon observed in the FEL oscillator experiment at the Los Alamos National Laboratory--the "walking mode"--which may be evidence for a refractive bending of the light in a cavity by the effective refractive index of the electron beam. This experiment will be described later (Sec. VII). 
In this paper we will first describe gain guiding, both for a quadratic medium and for a step-profile sptical fiber with a complex index of refraction. Gain guiding is (usually) the dominant form of optical guiding in the exponential gain regime of an FEL. Although gain guiding has not been observed experimentally in an FEL, it should be uncontroversial; gain guiding is the inevitable consequence of transversely localized gain comblned with diffraction. We then describe refractive guiding, relying primarily on the optical fiber analogy. Some numerical simulations are used to fllustrate optical gulding, and in particular refractive gulding in the absence of gain (after saturation in an untapered wiggler amplifier).

Gain and refractive guiding both coniribute to optical guiding in the exponential gain regime of the FEL. In this regime, nearly exact analytical treatments with diffraction effects fully included are possible. Several treatments are described in Sec. V.

In simulations it is possible to separate the effects of gain and refractive guiding. The technique for doing this is described in Sec. VIII, where the role of refractive gutding alone in a long tapered wiggler amplifter is adumbrated.

In this paper we will occasionally use the single-particle equations of motion, averaged over a wiggler perlod, with longitudinal space-charge forces ignored (i.e., the equations for a Compton-regime FEL). These equations were developed by KMR, ${ }^{6}$ by Prosnitz, Szoke, and Nell, 7 and by Colson. ${ }^{14}$ We repeat them here for reference. The longitudinal equations of particle motion are written for the Lorentz factor of an electron $\gamma_{j}$ and 1 ts normalized longitudinal position $\theta_{j}$, defined by

$$
\theta_{j} \equiv\left(k+k_{w}\right) z_{j}-\omega t
$$


where $k$ and $\omega$ are the wavenumber and frequency of the signal, and $k_{W}$ is the wiggler wavenumber. The equation for electron energy is

$$
\frac{d y_{j}}{d z}=-\frac{a_{w} f_{B} e_{s}}{y_{j}} \sin \psi_{j},
$$

where $\psi_{j} \equiv \theta_{j}+\phi$, with $\phi$ the phase of the signal electric field, is the phase of an electron in the ponderomotlve potential well, $a_{w}$ is the almensionless r.m.s. vector potentlal of the wiggler magnetic field:

$$
a_{w} \equiv \frac{e B_{w}}{\sqrt{2 m c} k_{w}}, \quad(1 \text { inear wiggler) }
$$

$e_{s}$ is the normalized r.m.5. electric field:

$$
e_{s} \equiv \frac{e E_{s}}{\sqrt{ } 2 m c^{2}}, \quad \text { (linear polarization) }
$$

with dimensions of inverse length, and $f_{B}$ is a difference of Bessel functions ${ }^{14}$ that represents a reduction of coupling between electrons and light in a linear wiggler caused by the osclilatory longltudinal motion of the electrons. For a helfcal wiggler and circular polarization, the factors of $\sqrt{2}$ are dropped (r.m.s and peak values are Identfcal), and $f_{\theta}=1$. We will use MKS units timroughout this paper.

The equation for $\theta_{j}$ is

$$
\frac{d \theta}{d z}=k_{w}-\frac{k}{2 \gamma_{j}^{2}}\left(1+a_{w}^{2}-2 a_{w} f_{B} a_{s} \cos \psi_{j}+\gamma^{2} \beta_{\perp j}^{2}\right) \text {, }
$$

where $a_{s} \equiv e_{s} / k$, and $\gamma \beta_{1 j}$ is the normalized perpendicular momentum associated with the betatron motion of the electron, averaged over a wiggler 
period. Including the $Y \beta_{1 j}$ terms permits us to incorporate electron beam emittance--one of the most important two-dimensional effects in an FEL--into the discussion. The FEL resonance, or synchronism, condition follows directly from Eq. (7) and the condition that $d \theta_{j} / d z \simeq 0$ :

$$
\lambda=\frac{\lambda_{w}}{2 \gamma_{j}^{2}}\left(1+a_{w}^{2}+\gamma^{2} \beta_{1 j}^{2}\right)
$$

Finally, the field equation for the complex amplitude $e_{s}$, derlved by assuming that the transverse current is periodic over many wavelengths and determining the Fourler component at $\omega$ and $k$, is

$$
\frac{d e_{s}}{d z}=\frac{1}{2 k} \nabla_{1}^{2} e_{s}+\frac{e z_{0}}{2 m c^{2}} a_{w} f_{B} j\left\langle\frac{e^{-1 \theta_{j}}}{Y_{j}}\right\rangle \text {, }
$$

where $Z_{0}$ is the impedance of free space, $J$ is the current density, and the angle brackets denote an average over electrons. The transverse gradient term in Eq. (9), $\nabla_{1}^{2} e_{s}$, is responsible for diffraction effects.

\section{Gain guiding}

Gain guiding should occur in any amplifying medium when the gain medium is transversely locailzed. Light is amplified by the medium as it simultaneously diffracts away from the medium (because of the localization). Propagation of the light with a constant transverse profile [e.g., $e_{s} \propto e_{s}(r) \exp (i n z)$, with $e_{s}(r)$ independent of $z$ ] is possible when gain is strong enough to balance (or overbalance) diffraction. Propagation with a constant transverse profile can be described as self-similar 
propagation of light; the profile at any $z$ is just a constant times the profile at any other $z$. Even with a constant transverse profile, power propagates away from the gain medium; phase fronts are therefore necessarily curved in gain gulded light.

The self-similar propagation of light in a medium with a quadratic gain profile

$$
\alpha=\alpha_{0}\left(1-\frac{r^{2}}{a^{2}}\right)
$$

has been analyzed by Kogelnik ${ }^{4}$ and experimentally demonstrated In Ref. 15. The gain $\alpha$ here is defined by the relation $|E| \propto \exp (\alpha z)$ for a uniform medium (no diffraction effects). For this simple case, the lowest order mode for the self-simllar light profile is exactly Gausslan chigher order modes are the Gauss-Laguerre modes of paraxlal diffraction theory).

The modes for gain guiding by a step-profile index fiber, for which

$$
\begin{array}{ll}
\alpha=\alpha_{0} & ; r<a \\
\alpha=0 & ; r>a
\end{array}
$$

i.e., the gain is uniform but localized to within the fiber radius a, are more complicated.

The simplest derivation of the modes for either form of gain gulding utilizes the paraxial wave approximation, wh!ch is the approximation that the amplitude and phase of the light vary slowly on the scale of an optical wavelength. The electric field of the light can then usefully be written

$$
E(x, y, z, t)=\operatorname{Re}\left[\mathscr{E}(x, y, z, t) e^{i(k z-\omega t)}\right] \hat{p},
$$

where $\hat{p}$ is the unit polarization vector [e.g., $\hat{p}=\hat{x}$ for linear polarization, $\hat{p}=(x+i y) / \sqrt{ } 2$ for circular polarization $]$, and $k \equiv \omega / c$. The complex 
amplitude $\mathscr{E}$ varies slowly in space and time; the rapid oscillations are contained entirely in the $\exp \{1(k z-\omega t)\}$ term. The wave equation for $E$,

$$
\nabla^{2} E-\mu_{0} \varepsilon_{0} \frac{\partial^{2} E}{\partial t^{2}}=\hat{p} \cdot(\text { source terms), }
$$

simplifies to

$$
2 i k \frac{\partial \mathscr{E}}{\partial z}+\nabla_{\perp}^{2} \mathscr{E}=e^{-1(k z-\omega t)} \hat{p} \cdot(\text { source terms) }
$$

when the complex amplitude $\mathscr{E}$ is independent of $t$ at fixed $z$, and the second derivatives of $\mathscr{E}$ with respect to $z$ can be neglected in comparison to $k$ times the first derivative:

$$
\left|\frac{\partial^{2} \mathscr{E}}{\partial z^{2}}\right| \ll k\left|\frac{\partial \mathscr{E}}{\partial z}\right| \text {. }
$$

A gcod discussion of the paraxial approximation, with a much more rigorous approach to the approximations involved, is contained in Ref. 16.

For a gain or refractive guiding by a medium characterized by a complex Index of refraction $n$, the right-hand side of Eq. (14) becomes

$$
e^{-1(k z-\omega t)} \hat{p} \cdot(\text { scurce terms })=-k^{2}\left(n^{2}-1\right) \mathscr{E} .
$$

The relation between gain $\alpha$ and complex index $n$ is

$$
\alpha=-k \operatorname{Im}(n)
$$

for $|\operatorname{Re}(n)-1| \ll 1$.

The assumption that the light is guided by the medium amounts to requiring that 


$$
\frac{\partial \mathscr{E}}{\partial z}=\ln \mathscr{E}
$$

for some constant $n$, independerit of $x, y$, or $z$. Then for gain guiding, the paraxial wave equation reduces to

$$
\nabla_{1}^{2} \mathscr{E}-2 k(\eta+1 \alpha) \mathscr{E}=0
$$

The lowest order mode for an axisymmetric medium is itself axisymmetric, so Eq. (19) can be rewritten

$$
\frac{1}{r} \frac{\partial}{\partial r} r \frac{\partial}{\partial r} E-2 k(n+1 \alpha) \mathscr{E}=0 .
$$

For a quadratic gain medium, with a given by Eq. (10), the solution to this equation is

$$
\mathscr{E}=\mathscr{E}_{0} \mathrm{e}^{i k r^{2} / 2 q}
$$

where $q$ is a complex radius of curvature for the guided mode, and is to be determined from Eq. (20). I: i: straightforward to show that

$$
q^{2}=-1 \frac{k_{a}^{2}}{2 \alpha_{0}} .
$$

Knowing $q$, the propagation constant $r$, can be found from

$$
\eta=i\left(\frac{1}{q} \cdots \alpha_{0}\right)
$$

The parameter $x_{0}$ characterizes the intrinsic gain of the medium; $-\operatorname{Im}(n)$ is the actual gain in the presence of diffraction:

$$
-\operatorname{Im}(n)=\alpha_{0}-\operatorname{Re}\left(\frac{1}{q}\right)
$$


As a consequence of this, gain exactly balances diffraction $[-\operatorname{Im}(\eta)=0]$ when $\alpha_{0}=1 / \mathrm{ka}^{2}$; any larger intrinsic gain $\left(\alpha_{0}\right)$ produces net gain $[-\operatorname{Im}(\eta)>0]$.

The complex radius of curvature $q$ is a standard parameter in the well-developed paraxial theory for the vacuum diffraction of light (see, for example, Kogelnik ${ }^{4}$ ). In terms of a beam spot size $w$ and a radius of curvature $R$ for phase fronts,

$$
\frac{1}{q}=\frac{1}{R}+\frac{2 \mathfrak{i}}{k w^{2}}
$$

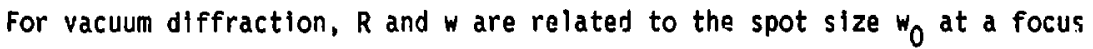
and distance $z$ from a focus by Eq, (2) and

$$
R=z\left[1+\frac{z_{R}^{2}}{z^{2}}\right],
$$

where the Rayleigh range, $z_{R}$, is defined by Eq. (1). A useful feature of the complex radius of curvature $q$ is that one can write very simpiy (for vacuum propagation)

$$
q=z-i z_{R}
$$

The expression (22) for $q$ in a quadratic gain medium then indicates the significant property that the phase fronts of the gain-guided light are curved at any point to appear as if they propagated in vacuum from a focus precisely one Rayleigh range behind the point. As we will see below, the phase fronts of refractively guided light are not curved at all; the phase front curvature is one potentially practical way to observationally (or numerically) distingtish gain from refractive guiding. 
The property that the effective focus is precisely one Rayleigh range behind the observation point is only true for gain guiding in a quadratic medium, unfortunately. It is approximately true in certain limits of gain guiding by a step-profile index fiber, as we now show.

For the gain profile of Eq. (11), the solutions to the paraxial-wave equation (20) become Bessel functions

$$
\begin{aligned}
& \mathscr{E}=\mathscr{E}_{0} J_{0}(\kappa r), \quad r<a \\
& \mathscr{E}=A \mathscr{E}_{0} K_{0}(\gamma r), \quad r>a
\end{aligned}
$$

where $\kappa$ and $\gamma$ are complex parameters that characterize the propagation, $I_{0}$ is a Bessel function, and $K_{0}$ is a modifled Bessel function. The symbol $\gamma$ has been used previously in this paper to denote the Lorentz factor of an electron; we reuse it here to permit a link to the theory for weakly guiding optical fibers developed in, for example, Marcuse. ${ }^{17}$

From the wave equation,

$$
\begin{aligned}
& \kappa=\left[-2 k\left(\eta+1 \alpha_{0}\right)\right]^{1 / 2}, \\
& \gamma=[2 k \eta]^{1 / 2} .
\end{aligned}
$$

The choice of Bessel functions, $J_{0}$ and $K_{0}$, was determined by the boundary conditions (finite $\mathscr{E}$ ) at $r=0$ and $r+\infty$. Those boundary conditions also impose the constraint that $\operatorname{Re}(\gamma)>0$, since $K_{0}(\gamma r) \propto \exp (-\gamma r) / J r$ as $r \rightarrow \infty$. The condition that the phase velocity of the 1 ight be directed away from the fiber constrains $\operatorname{Im}(\gamma)$ to be $<0$. The propagation constant $\eta$, and hence $\kappa$ and $\gamma$, are determined by the boundary conditions on $\mathscr{E}$ at $r=a$ :

$$
\begin{aligned}
& \text { continuous } \mathscr{E} \rightarrow J_{0}(k a)=A K_{0}(\gamma a) . \\
& \text { continuous } \frac{\partial \mathscr{E}}{\partial r} \rightarrow \kappa J_{1}(\kappa a)=A \gamma K_{1}(\gamma a) . \\
&-12-
\end{aligned}
$$


El.iminating $A$, we find a dispersion relation for $x$ and $y$ :

$$
\frac{k J_{1}(k a)}{J_{0}(k a)}=\frac{\gamma K_{1}(\gamma a)}{K_{0}(y a)}
$$

with

$$
k^{2}+\gamma^{2}=-2 i k \alpha_{0}
$$

Since $x$ and $y$ are complex, it is necessary to solve the dispersion relation numerically. Here, we are primarlly interested in the phase curvature of the solution, and the actual galn, $-\operatorname{Im}(n)$, as a function of $k$, $a$, and $\alpha$. We can parametrize the quantities of interest by

$$
v^{2}=\left(n^{2}-1\right) k^{2} a^{2} \text {, }
$$

which, for a real index of refraction, is known as the fiber parameter. 17 For pure gain,

$$
v^{2} \simeq-412{ }_{R B}^{\alpha} O
$$

where $z_{R B}$ is a Rayleigh range obtained from the fiber radius:

$$
z_{R B}=\frac{k a^{2}}{2}=\frac{\pi a^{2}}{\lambda}
$$

We determine a complex radius of curvature $q$ by equating

$$
-\frac{k^{2} r^{2}}{4}=\frac{i k r^{2}}{2 q}
$$

to obtain

$$
q=-\frac{4 i}{(\kappa a)^{2}} z_{R B}
$$


and sc

$$
-\frac{4 i}{(\kappa a)^{2}}=\frac{z}{z_{R B}}-i \frac{z_{R}}{z_{R B}} \text {. }
$$

where now $z_{R}$ is a Rayleigh range determined from the phase front curvature and electric field gradient near the axis. From Eq. (41) we can extract the ratio $z / z_{R}$, which determines the location of the virturl focus of the 1ight. The actual gain, $-\operatorname{Im}(n)$, can be obtained from Eq. (24), and written

$$
-\operatorname{Im}(n) z_{R B}=\alpha_{0} z_{R B}+\frac{1}{4} \operatorname{Im}\left(\kappa^{2} a^{2}\right)<\alpha_{0} z_{R B} .
$$

In FIg. 2 we plot $z / z_{R}$ as a function of $\operatorname{Im}\left(V^{2}\right)<0$; recall that a more negative $\operatorname{Im}\left(V^{2}\right)$ corresponds to stronger gain. We see from the figure that (a) no gain-guided solution exists for $\operatorname{Im}\left(V^{2}\right)>-1.87$, and $(b)$ the virtual focus is one Rayleigh range behind the observation point only near the cutoff, i.e., for $-3.0<\operatorname{Im}\left(v^{2}\right)<-1.87$. As gain increases, the virtual focus moves closer to the point of observation.

Curved phase fronts can therefore be considered indicative of gain guiding, but the location of the virtual focus cannot be used to disentangle gain and refractive guiding.

\section{Refractive quiding}

The equations used in the previous section to exiamine the properties of gain guiding can also be used to examine refractive guiding, with the simple substitution

$$
\alpha \rightarrow i k \equiv i k(n-1) \text {, }
$$


where $n$, and hence $k, \gamma, \eta$, and $v^{2}$, are all purely real numbers, and $q$ is purely imaginary.

The derivations above can all be duplicated, to obtain (for a step-index fiber)

$$
\begin{aligned}
& k=[2 k(k-\eta)]^{1 / 2}, \\
& y=(2 k n)^{1 / 2}, \\
& V^{2}=2 k K a^{2}=4 k z_{R B} .
\end{aligned}
$$

The dispersion relation for $k$ and $y$, Eq. (34), stlll holds. There exlsts a solution to that dispersion relation. With the subsidiary condition that $\left(x^{2}+\gamma^{2}\right)=v^{2}$, for any $v^{2}$, indicating that this lowest order mode does not have a cutoff--it can propagate for an arbitrarily small $n-1$. For a medium with a quadratic index profile,

$$
K=k_{0}\left(1-\frac{r^{2}}{a^{2}}\right),
$$

the complex radius of curvature $q$ becomes

$$
q=\left(\frac{k_{a}^{2}}{2 k_{0}}\right)^{1 / 2}
$$

In both cases, phase fronts of the light are exactly flat, Indicating (among other things) that power is not propagating away from the medium. For this case, the self-similar profile is possible because refraction is balancing diffraction exactly, not because gain is replenishing power lost to diffraction. 


\section{The index of refraction in an FEL}

In, for example, a glass with a real index of refraction $n>1$, the index of refraction arises because light, propagating through the glass, excites atomic dipoles which reradiate $90^{\circ}$ out of phase with the incident light. If the reradiated light is precisely $90^{\circ}$ out of phase, it only shifts the phase of the incident light, with no loss or gain. The propagation of light in $\because \cdots \quad r$ the guiding of light by a conventional optical fiber, can be ely described by summing the incldent light and the reradiated light from each atomic dipole; since this would obviously be a tedious calculation, the effect of adding reradiated light is commonly treated by a phenomenological index of refraction $n$.

The important point in that brief description of a refractive index--at least for understanding the origin of the index in an FEL--is that the index can be used to represent the effect of light radiated by many microscopic dipoles. For understanding the propagation alone, the origin of the dipole radiation does not matter; the dipoles could be microscopic antennas operated by excitable demons without changing the description of propagation. In particular, for the FEL, the dipoles are individual bunches in the electron beam, moving in the magnetic field of the wiggler. The bunches are approximately the size of an optical wavelength, and are formed by the gain mechäntsm of the FEL--fiì a tapered wiggier, the uunches are the eiectrons trapped in ponderomotive potentials.

We can clarify the mechanism of refractive guiding with a simple example of this dipole description, treating the electron beam as a linear array of dipoles with a periodic, time-independent density $\propto \sin \left(k_{b} z\right)$ and periodic, 
time-dependent phasing $\propto \cos \left(k_{d} z+\omega t\right)$. Then the dipole moment $d \vec{p}$ of the array within an infinitesimal distance $d z$ is

$$
d \vec{p}=\vec{p}_{0} \sin \left(k_{b} z\right) \cos \left(k_{d} z+\omega t\right) .
$$

This description corresponds to a bunched electron beam wiggling in a frame moving with the average velocity of the electron beam (the beam frame). The dipole density modulation is the electron bunching, and the dipole phasing and motion is provided by the wiggler which, in the beam frame, is a backward propagating wave $\propto \cos \left(k_{d} z+\omega t\right)$.

The simplest gulded wave can be constructed by integrating the radlation field from the line of dipoles described by Eq. (49). From a single dipole dp:

$$
d \vec{A}(\vec{r})=-i k d \vec{p} \frac{e^{i k r}}{r}
$$

where $\vec{A}(r, t)$ is the vector potential of the radiation in the Lorentz gauge and $k=\omega / c$. An exp $(-1 \omega t)$ dependence is assumed, as in Jackson. ${ }^{18}$ Integrating over all dipoles, one readily finds that at an observation polnt $\left(r_{0}, z_{0}\right)$,

$$
\begin{aligned}
\vec{A}\left(r_{0}, z_{0}\right) & =-i k \vec{p}_{0}\left\{k_{0}\left[\left(k_{+}^{2}-k^{2}\right)^{1 / 2} r_{0}\right] \sin \left(k_{+} z_{0}+\omega t\right)\right. \\
& \left.+k_{0}\left[\left(k_{-}^{2}-k^{2}\right)^{1 / 2} r_{0}\right] \sin \left(k_{-} z_{0}-\omega t\right)\right\}
\end{aligned}
$$

with $k_{ \pm} \equiv k_{b}=k_{d}>k$; no approximation beyond the dipole assumption is made in obtaining Eq. (51). The first term on the right-hand side of Eq. (51) represents the coherent superposition of a pattern with a spatial 
wavenumber $k_{b}+k_{d}$ very much greater than $k=\omega / c$ [for $F E L$ parameters, in the beam frame, $\left.k_{b}+k_{d} \simeq 3 k\right]$. The superposition therefore is primarlly destructive interference, producing a very rapid transverse decay of this field component, on a scale less than a wavelength of light; the pattern also has a backward phase velocity in the beam frame. This field component is unimportant for an FEL and we henceforth isnore it.

The other (second) term on the right-hand side of Eq. (51) represents the interesting guided solution. Power propagates entirely in the z direction, with exponential decay of the radiation with radius; the decay can be quite slow for $k_{-}^{2}-k^{2} \ll k^{2}$. Note that the guided solution, in amplitude, phase, and radial profile, is unique, as required by the linearity of Maxwell's equations for fixed source. The unique, guided solution also exists for any uniformly bunched beam. A criterion for optical guiding in an FEL to be important is therefore very difficult to construct--there will be some guided solution for any bunched beam, and the significant question becomes whether or not that guided solution is interesting.

It could be argued that the fields in Eq. (51) do not represent a true guided solution, that the radial profile is merely a result of destructive interference between dipoles that are radiating slightly out of phase. The radial profile is indeed due to destructive interference, at large $r$, between the radiation fields of many dipoles, but the destructive interference is precisely the origin of refractive guiding, either in an FEL or in an optical fiber. The guided solution represented by Eq. (51) corresponds very closely to the expected behavior of light in an FEL amplifier with an untapered wiggler, after saturation. Fig. 3 illustrates, from numerical simulation, that expected behavior. The light builds up in the exponential gain regime 
until saturation; after saturation, the light intensity oscillates somewhat (the bunching in the beam is not precisely stationary), but the predominant feature is the guiding of the light in the absence of gain. The guiding lllustrated in the figure can be described by the guided solution of Eq. (51).

\section{Guiding in the exponential gain regime of an FEL}

- Optical guiding in an FEL has been most completely analyzed in the exponential gain regime, in which the signal strength is weak enough that perturbations to the electrons' $\gamma$ and $\psi$ can be treated as llnear quantitles. To treat this regime, the dynamics of the electrons [Eqs. (4) and (7) ] must be followed with the fleld equation; the electrons provide the source term in Eq. (13). That source term is no longer linearly proportional to $\mathscr{E}$, because the dipole motion that produces the source term is not the response of atomic dipoles to the incident electric fleld; instead, it is the motion of electron bunches in the wiggler fleld. That physical difference does not introduce a significant difference in the propagation equations.

The linearization of particle and fleld equations is described in Refs. 10, 11, and 19. Ref5, 10 and 11 Ignore transverse varlations in the electron beam, implicitly assuming that the properties of the electron beam are constant within a radius a; this assumption yields a very strong analogy to the optical fiber, including the appearance of the fiber dispersion relation, Eqs. (34) and (35) [with complex $k$ and $y$ ], above. The fiber dispersion relation is supplemented by a cubic equation, which contains the electron dynamics (including energy spread or emittance).

$X i e$ and Deacon ${ }^{19}$ treat the transverse variation of the electron beam and optical mode in a very different fashion: the linearized field equation 
is radially integrated with a Gaussian, $\exp \left(-p r^{2}\right)$, to obtain a cublc equation for $n$ that contains the free parameter $p$. By analogy with an optical fiber, in which the lowest order mode maximizes the propagation constant, Xie and Deacon argue that $p$ is determined by maximizing the growth rate, $-\operatorname{Im}(n)$. This varlational condition permits them to eliminate $p$ from the cubic, ylelding a single cubic equation for the FEL growth. Their method is useful only for an electron beam with no energy spread or emittance; if one includes energy spread and emittance, a supplementary equation must simultaneously be solved.

\section{Applicability of Kramers-Kronig relations}

The possibility of refractive guiding in an FEL may at first glance be surprising; as mentioned earlier, the Kramers-Kronig relations usually (in a normal laser medium) require that the real part of the index of refraction of a gain medium vanish at peak gain.

The Kramers-Kronig relations relate the real and imaginary parts of the one-sided (in time) Fouriar transform of a linear response, such as a conductivity or permittivity. Causality alone requires that the Fourier transform be one sided, and the one-sided nature of the transform ensures that the transformed function is analytic in the upper half complex w plane. Then the real and imaginary parts of the transformed function form a Hilbert transform pair [see, for example, Ref. 20]. If then the gain is a symmetric function of frequency around a central $\omega_{0}, \operatorname{Re}\left[n\left(\omega_{0}\right)\right]=0$.

A more detailed discussion here of the derivation of the Kramers-Kronig relations is not warranted, because the key word above is "linear". For an FEL beyond saturation of exponential gain, the response function is very 
nonlinear. For example, we can look at a short section of wiggler with a long, bunched electron beam moving through it. The field at the beginning of the short section of wiggler is $\mathscr{E}_{\text {in }}$, and at the end is $\mathscr{E}_{\text {out }}$. Associated with the change from $\mathscr{E}_{\text {in }}$ to $\mathscr{E}_{\text {out }}$ is some gain and some phase shift. Are the phase shift and gain related by the Kramers-Kronig relations? If one could write

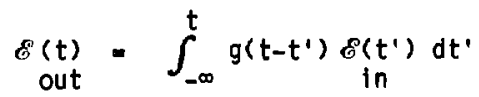

where $g$ is any function that depends only on $t-t^{\prime}$, then indeed the gain and phase shift would be related by the Kramers-Kronig relations. In fact, though, we cannot write any equation $11 \mathrm{ke} E q$. (52), except in the exponential gain regime. In the opposite limit, in which the electron bunches do not respond to changes in $\mathscr{E}_{i n}(t)$, but instead radiate a fixed $\Delta \mathscr{E}(t)$, we can write

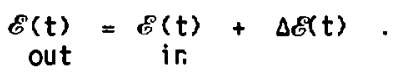

The gain and phase shift are very nonlinear, and no general reiation between them can be derived.

In the exponential gain regime, the Kramers-Kronig relations must hold; the response is by definition linear in the input signal. Figs. 4 and 5 lllustrate the gain and phase shift expected for our 21- $\mu \mathrm{m}$ FEL. The curves are calculated from the cubic equation for exponential gain that has been derived in Ref. 11. The characteristic form required by the Kramers-Kronig relations is evident in the figures: the gain is posftive over a narrow range of wavelengths, and the phase shift monotonically decreases over that range. The phase shift does not vanish at the peak of the gain curve, however, because of the asymmetry in the gain curve.

$$
-21 \text { - }
$$


VII. Experimental evidence for refractive guiding?

Experimental confirmation of the possibility of bending an optical beam with a bunched electron beam may have been achieves in the Los Alamos oscillator experiment. ${ }^{21,22}$ The experimental observation is of 400-ns modulation of the output power from the oscillator, when the electron beam is misaligned with the axis of the optical cavity, and when the electron beam macropulse has ceased, so that the high-Q cavity is ringing down. Refs, 21 and 22 interpret the modulation to be evidence for a bent equilibrium optical mode during lasing, as lllustrated in Flg. 6 . When the light interacts with the electron beam on the forward pass, it is bent by the refractive index of the electron beam; on the backward pass, the light does not interact with the beam, and travels in a straight line. Since the electron beam is not ailigned with the axis of the resonator, an equilibrium mode represented by the optical rays in Fig. 6 can be established.

After the electron beam macropulse shuts off and lasing ceases, this bent mode is no longer an equilibrium mode. Instead the mode "walks" up and down across the mirrors. If the output coupler is, for example, a small hole in the center of the right-hand mirror, then as the mode walks up and down, the output power will be modulated with the period of the walking--as observed.

The identification of refractive guiding as being responsible for the bending of the light in the cavity comes from numerical simulation. As described below (Sec. VIII), it is possible to shut off refractive guiding in simulations. When this is done, ${ }^{22}$ the equilibrium optical mode no longer shows the bending of Fig. 6 during lasing. 


\section{Importance of refractive guiding for an amplifier}

He can examine the role of refractive guiding in our $21-\mu m$ simulations with two FRED runs, one in which the full set of FEL equations is used, the other in which refractive guiding is artificlally suppressed. The suppression is done by zeroing the part of the source term in the FEL equations that is $90^{\circ}$ out of phase with the electric field; this ensures that the FEL produces gain with no phase shift. $\operatorname{Re}(n)-1=0$. Ail exce'lent check on the modified coding is provided by energy conservation; since energy is conserved with or without the modifications used to null refractive guiding, the proper component of the source term has been zeroed.

FRED follows a slowly-varying, complex field amplitude $e_{s}(r, z)$. The source term for $\partial e_{s} / \partial z$ is proportional to $i\langle\exp (-1 e) / \gamma\rangle[E q, 9]$, where $\theta_{j}$ for the $j^{\text {th }}$ electron is defined in Eq. (3). Gain is produced by the source term in phase with $e_{s}$, and a phase shift is produced by the source term out of phase with $e_{s}$. Removing the phase shift without affecting gain (and therefore without destroying energy conservation) involves the replacements:

$$
\begin{aligned}
& \cos \theta_{j}+\left[\sin \theta_{j} \cos \phi+\cos \theta_{j} \sin \phi\right] \sin \phi, \\
& \sin \theta_{j}+\left[\sin \theta_{j} \cos \phi+\cos \theta_{j} \sin \phi\right] \cos \phi,
\end{aligned}
$$

for each electron, just before constructing the field source. As before, $\phi$ is the phase of the local electric field. A switch was added to FinE to make these replacements, and thereby renove refractive guiding.

Figure 7 compares the power in the wiggler vs $z$, with and without refractive guiding. Without refractive guiding (gain guiding alone), the 
output power is reduced by about a factor of three. A more dramatic effect is shown in Fig. 8 where the spot size, determined from the r.m.s. radius of the intensity distribution, is plotted vs $z$, with and without refractive guiding. Refractive guiding clearly makes a large difference in spot size.

A third difference is shown in Figs. 9 through 12, where the intensity profile (Figs. 9 and 10) and phase proflle (Figs. 11 and 12) at the output of the wiggler are shown, with (Figs. 9 and 11) and without (Figs. 10 and 12) refractive gulding. Without refractive guiding, the psak intensity is reduced by an order of magnitude, and the beam quality is very much worse: the beam with refractive guiding is nearly diffraction limited, as shown by the fit of intensity to a Gaussian profile in Fig. 3 and phase to a spherical profile in F:g. 11. Without refractive guiding, the fit is very much worse.

\section{Acknowledgements}

I am happy to acknowledge useful dicussions with $Y$. J. Chen and A. Sessler about many aspects of optical guiding. 
Table 1. Simulation parameters

Beam energy

Current

Emittance

(normalized edge)

Beam radius

Wavelength

Input power

Input spot radius

Wiggler period

Wiggler length

Initial magnetic fleld
$38 \mathrm{MeV}$

$1 \mathrm{kA}$

$0.1 \mathrm{rad}-\mathrm{cm}$

$0.26 \mathrm{~cm}$

$21 \mu \mathrm{m}$

$100 \mathrm{~kW}$

$0.25 \mathrm{~cm}$

$5.5 \mathrm{~cm}$

$12 \mathrm{~m}$

$4.93 \mathrm{kG}$ 


\section{REFERENCES}

1. H. M. Fawley, D. Prosnitz, E. T. Scharlemann, Phys. Rev. A30 (1984) 2472.

2. E. T. Scharlemann, Journ. Applied Phys. 58 (1985) 2154.

3. E. T. Scharlemann and W. M. Fawley, SPIE 642 (1986) 2.

4. H. Kogelnik, Appl. Optics 4 (1965) 1562.

5. J. M. Slater and O. D. Lowenthal, Journ. Appl. Phys. 52 (1981) 44.

6. N. M. Kroll, P. L. Morton, M. R. Rosenbluth, IEEE Journ. Quantum Electronics QE-17 (1981) 1436.

7. D. Prosnitz, A. Szoke, and V. K. Ne11, Phys, Rev. A24 (1981) 1436.

8. P. Sprangle and C. M. Tang. Applled Physics Letters 39 (1981) 677 ;

C. M. Tang and P. Sprangle, in Physics of Quantum Electronics, Vol. 9 (Addison-Hesley.Reading, Mass., 1982) 627.

9. D. Prosnitz, H. M. Fawley, R. Gelinas, and S. Doss, in Physles of Quantum Electionics, vol. 9 (Addi son-Kesley,Reading, Mass., 1982) 1054.

10. G. T. Moore, Optics Communications 52 (1984) 46 .

11. E. T. Scharlemann, A. M. Sessler, and J. S. Hurtele, Phys. Rev. Lett. 54 (1985) 1925.

12. J. E. LaSala, D. A. G. Deacon, and J. M. J. Madey, in Free-Electron Lasers, Proc. 7th International Free-Electron Laser Conference, eds., E. T. Scharlemann and D. Prosn'tz (North Holland, Amsterdam, 1986) p. 262.

13. M. Cornacchia, J. Blsognano, S. Chattopadhyay, A. Garren, K. Halbach,

A. Jackson, K. J. KIm, H. Lancaster, J. Peterson, M. S. Zisman,

C. Pellegrint, and G. Vignola, op. cit. p.57.

14. W. B. Colson, IEEE Journ. Quantum Electronics QE-17 (1981) 1417.

15. L. Casperson and A. Yariv, Appl. Phys. Letters 12 (1968) 355.

16. J. H. Marburger, Prog. Quantum Electronics 4 (1975) 35.

17. D. Marcuse, Theory of Dielectric Optical Waveguides, (Academic, New York, 1974), p. $60 f f$.

18. J. D. Jackson, Classtcal Electrodynamtcs, (Włley, New York, 1962).

19. M. Xie and D. A. G. Deacon, in Free-Electron Lasers, Proc. 7th International Free-Electron Laser Conference, eds., E. T. Scharlemann and D. Prosriitz (North Holland, Amsterdam, 1986) p. 426. 
20. B. G. Levich, Theoretical Physics, (North Holland, Amsterdam, 1971) p. $540 f f$.

21. R. W. Warren and B. McVey, Bending and Focusing Effects in FEL Osciliators: I. Simple Models, preprint, to be published in the Proc. of the 8th Internationial Free-Electron Laser Conference, ed. M. Poole (North Holland, Amsterdam, 1987).

22. B. McVey and R. H. Warren, Bending and Focusing Effects in FEL Oscillators: II. Numerical Simulations, preprint, to be published in the Proc. of the 8th International Free-Electron Laser Conference, ed.

M. Poole (North Holland, Amsterdam, 1987). 


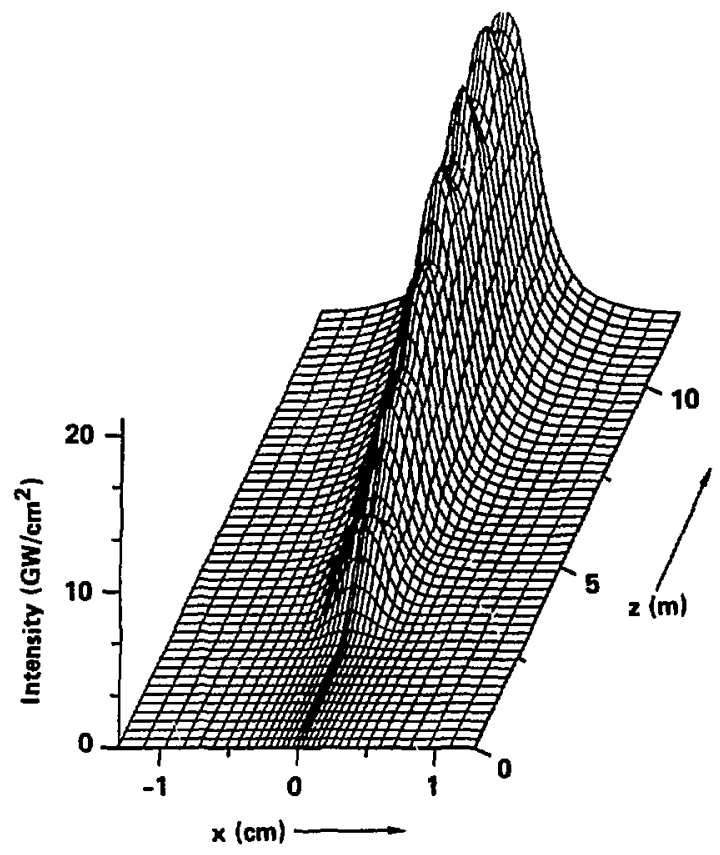

Fig. 1: An isometric plot of optical intensity vs $x$ and $z$ in the wiggler, for the amplifier described by Table 1. 


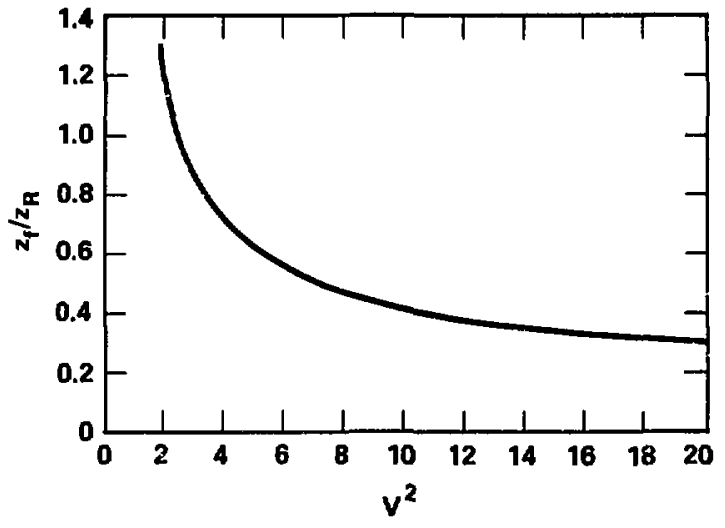

Fig. 2: The location of the virtual focus behind any point in a light proflle guided by a fiber with gain and no refraction, plotted as a function of $-\operatorname{Im}\left(V^{2}\right)$, where $V^{2}$ is the fiber parimeter. 


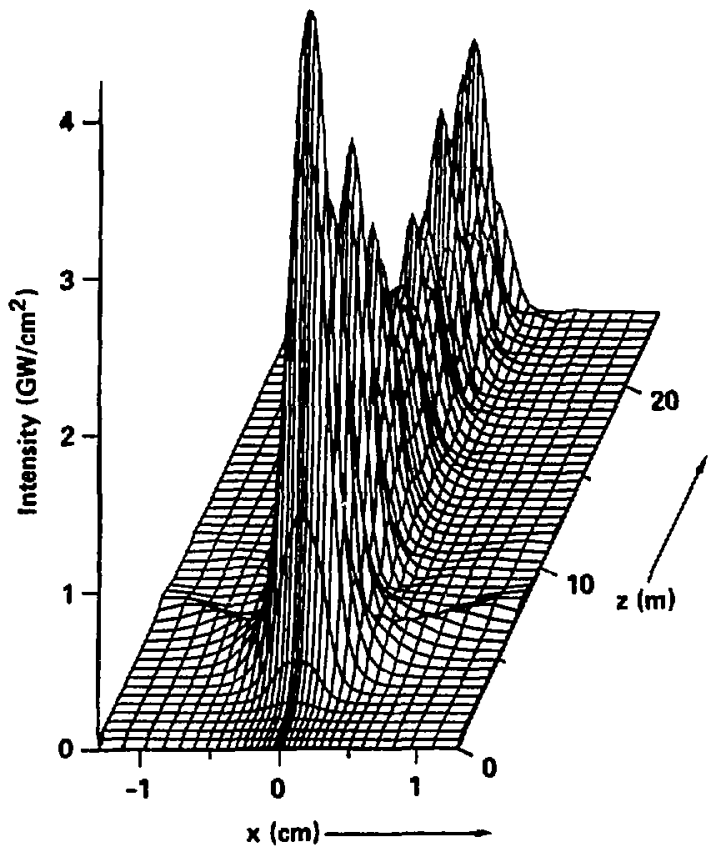

Fig. 3: An isometric plot of optical intensity vs $x$ and $z$ in an untapered wiggler, fllustrating refractlve guiding of the light past saturation. 


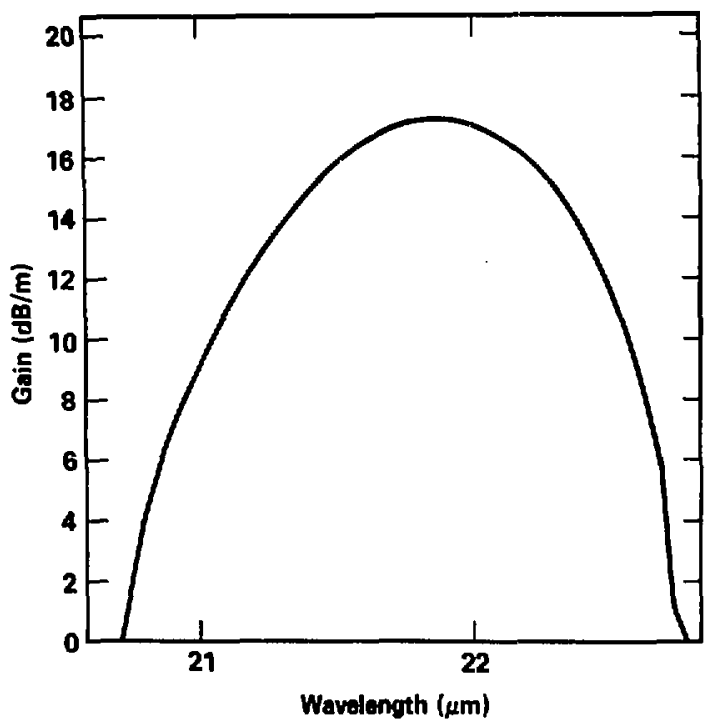

Fig. 4: Gain in $d B / m$ vs wavelength for the $21-\mu m$ FEL amplifier. 


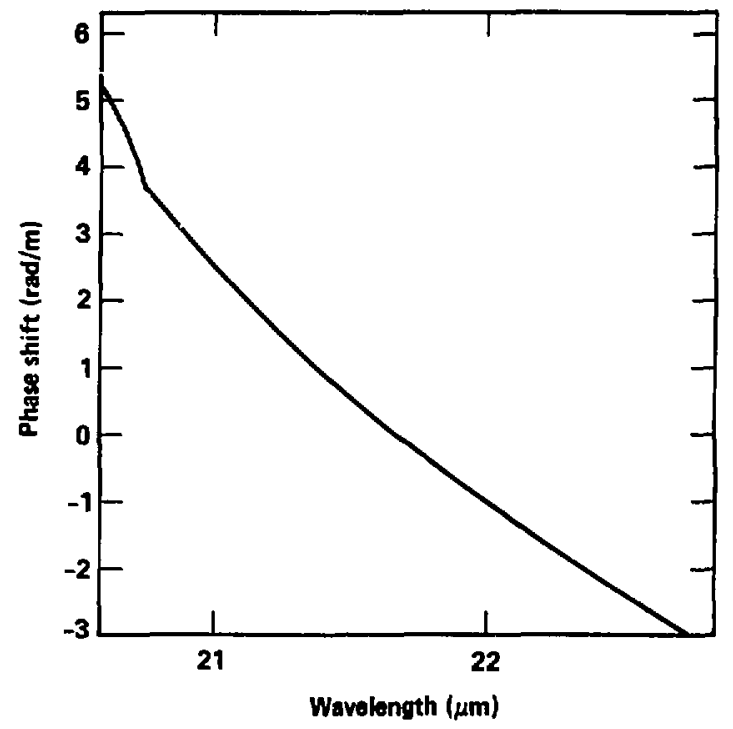

Fig. 5: Phase shift in rad/m vs wavelength for the 21- $\mu \mathrm{m}$ amplifier. 


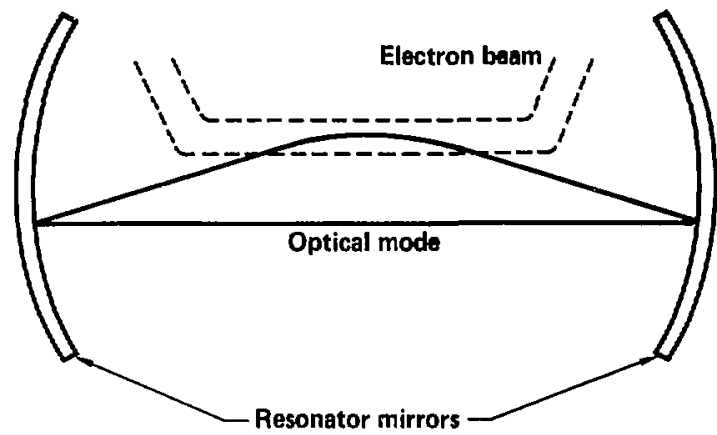

Fig. 6: An lllustration of a bent equilibrium optical mode in an oscillator, with the bending provided by the bunched electron beam in the wiggler. 


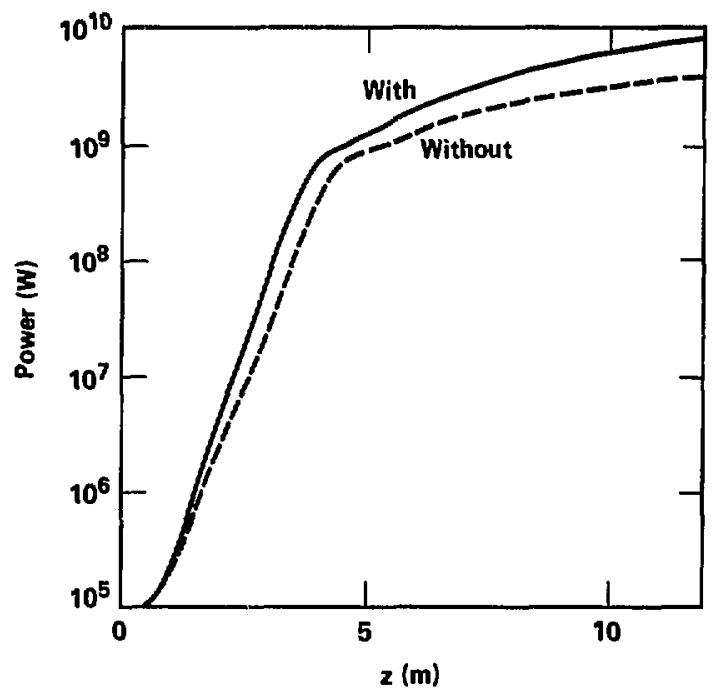

Fig. 7: Power v5 wiggler length for the 21-um FEL amplifier, with and without refractive guiding in the simulations. 


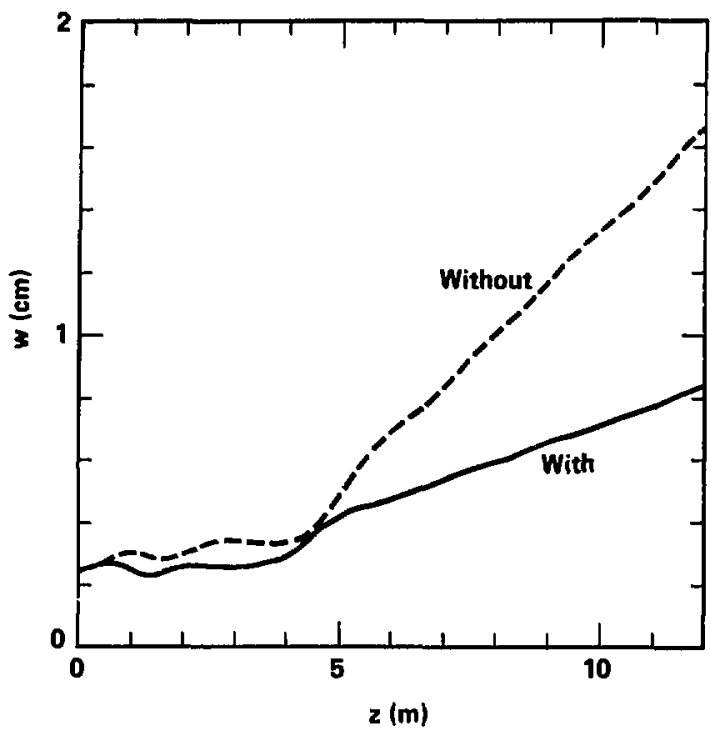

Fig. 8: Optical spot radius vs wiggler length for the 21- $\mu m$ amplifier, with and without refractive guiding. 


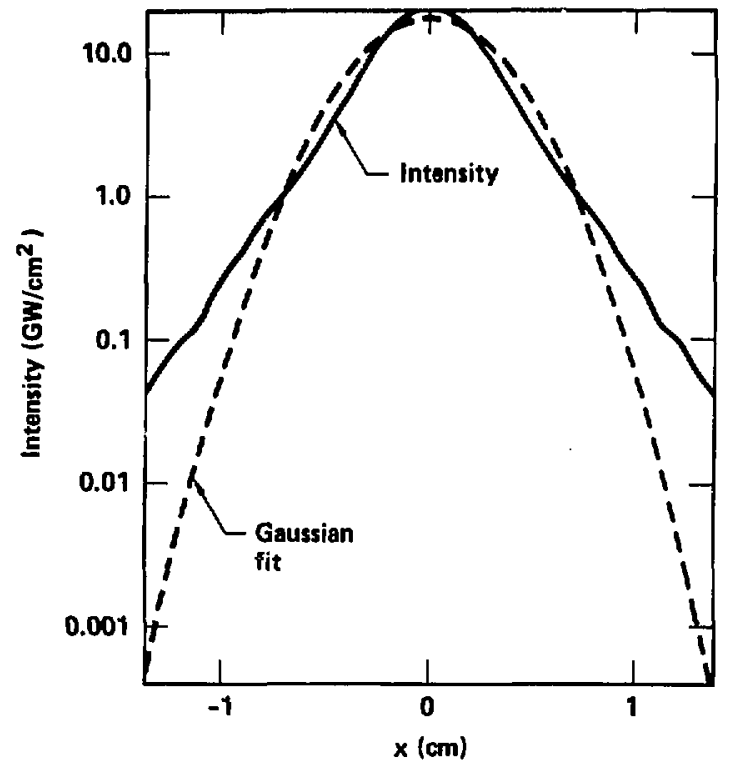

Fig. 9: Output intensity proflle of the 21-um amplifier. 


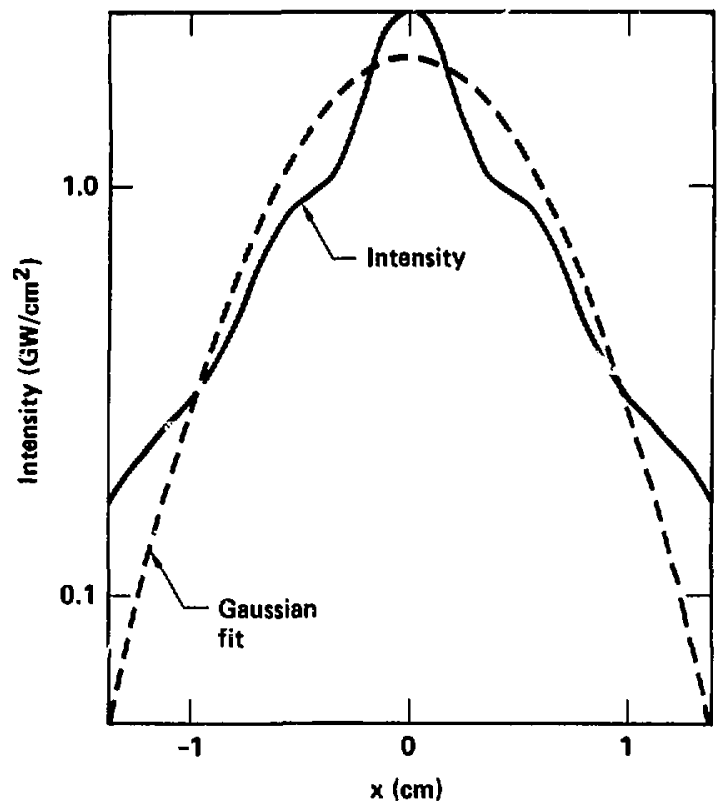

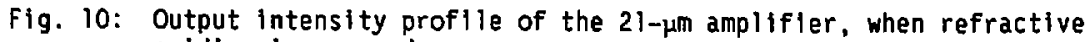
guiding is removed. 


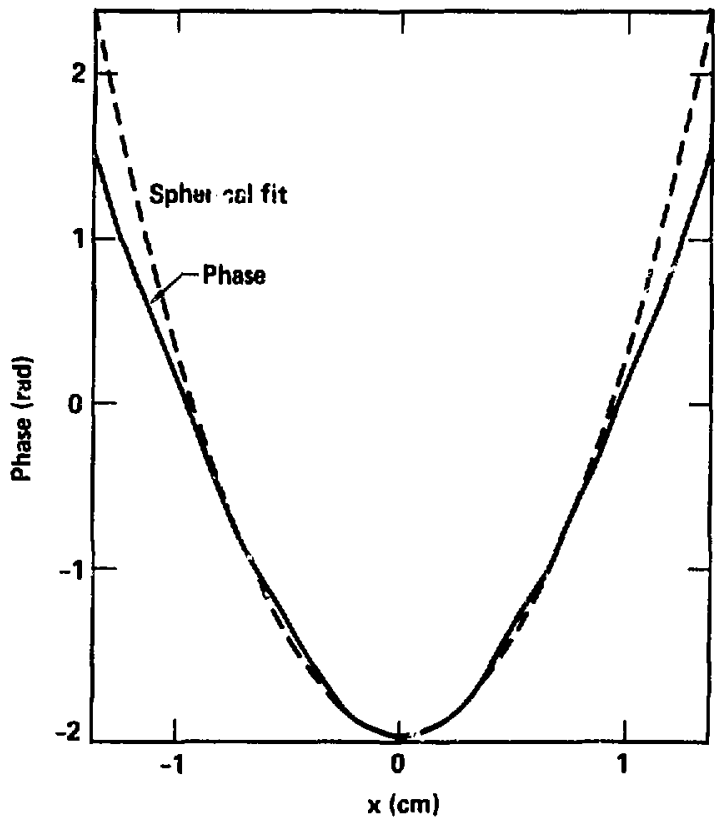

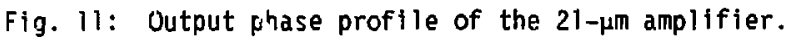




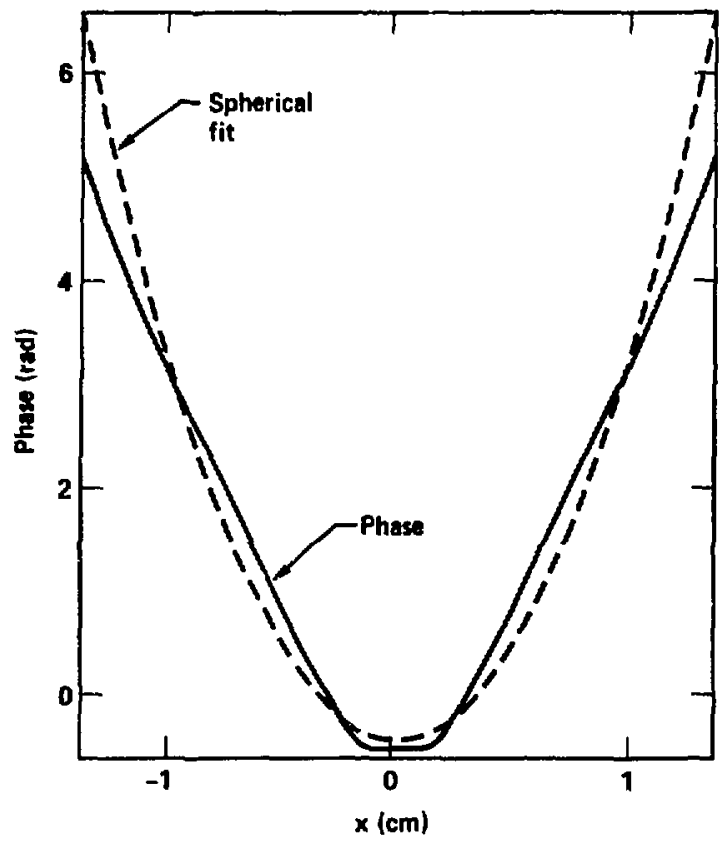

Fig. 12: Output phase profile of the 21-um amplifier, when refractive guiding is removed. 